Даус Юлия Владимировна, инженер отдела НИОКР, интеллектуальной собственности и трансфера технологий, ФГБОУ ВО «Санкт-Петербургский государственный агарный университет» (РФ, 196605 , г. Санкт-Петербург, Петербургское ш., д. 2), кандидат технических наук, тел. 8 (812) 476-2333, e-mail: zirochka2505@gmail.com.

Юдаев Игорь Викторович, проректор по учебной и воспитательной работе, ФГБОУ ВО «СанктПетербургский государственный аграрный университет» (РФ, 196605, г. Санкт-Петербург, Петербургское ш., д. 2), доктор технических наук, профессор, тел. 8 (812) 476-23-33, e-mail: etsh1965@mail.ru.

\title{
KINEMATICS OF MOTION OF THE MANIPULATING MECHANISM OF A ROBOTIC PROPROLLER DURING THE TECHNOLOGICAL PROCESS OF WEEDING
}

\author{
A. G. Ivanov, N. S. Vorob'yeva \\ Volgograd State Agrarian University, Volgograd, Russia
}

Received 10.09.2021

Submitted 15.11.2021

\section{Summary}

The article presents calculations of the movement of the working tool along a previously unknown and straight path, a comparative analysis was carried out, the result of which revealed that it is preferable to use a previously undefined trajectory for the movement of the working tool of a robotic weeding machine.

\begin{abstract}
Introduction. The article is devoted to the development of programmed movements of a working part, a robotic weeding machine in space by the method of kinematic synthesis. Object. The working part of the robotic weeding machine is used as a milling cutter with a vertical axis of rotation. Materials and methods. The solution to the problem of moving the working part (cutter) in space is divided into two stages. 1. The problem of positioning the manipulation mechanism (configuration problem) is solved, which provides the final position in the visual cutter of the weeder, determined by the coordinates of the weed, based on the optimization criterion. 2. After the configuration problem has been solved, the task is to find the motion of the executive links along the trajectory. The paper considers without inertial movement of the working part of a robotic weeding machine along two trajectories: 1 straight; 2 - undefined in advance. Results and conclusions. The problem of moving the working part (milling cutter) of a weeding machine when moving along a straight line and along a previously undefined trajectory according to the law of inertialess motion using an optimal positioning algorithm has been solved. A comparative analysis of the movement of the working part of the weeding machine showed that the elongation along the indefinite trajectory of the working part is insignificant (up to $6 \%$ ), and also that when the working part moves in a straight line, the rod of one actuator makes a reciprocating motion, which in turn negatively affects the drive. Therefore, it is preferable to use a previously undefined trajectory to move the weeding tool.
\end{abstract}

Key words: robotic weeding machine, technological process of weeding, working part, executive links, trajectory of movement, law of motion.

Citation. A. G. Ivanov, N. S. Vorob'yeva. Kinematics of motion of the manipulating mechanism of a robotic proproller during the technological process of weeding. Proc. of the Lower Volga AgroUniversity Comp. 2021. 4(64). 452-463 (in Russian). DOI: 10.32786/2071-9485-2021-04-44.

Author's contribution. All authors of this research paper have directly participated in the planning, execution, or analysis of this study. All authors of this paper have read and approved the final version submitted.

Conflict of interest. The authors declare no conflict of interest. 


\title{
***** H3BECTYЯ ***** \\ НИЖНЕВОАЖСКОГО АГРОУНИВЕРСИТЕТСКОГО КОМПАЕКСА: \\ НАУКА И ВЫСШЕЕ ПРОФЕССИОНААЬНОЕ ОБРАЗОВАНИЕ \\ КИНЕМАТИКА ДВИЖЕНИЯ МАНИПУЛЯЦИОННОГО МЕХАНИЗМА РОБОТИЗИРОВАННОГО ПРОПОЛЬЩИКА ПРИ ВЫПОЛНЕНИИ ТЕХНОЛОГИЧЕСКОГО ПРОЦЕССА ПРОПОЛКИ
}

\author{
А. Г. Иванов, ассистент \\ Н. С. Воробьева, кандидат технических наук, доцент \\ Волгоградский государственный аграрный университет, г. Волгоград
}

Дата поступления в редакцию 10.09.2021

Дата принятия к печати 15.11.2021

Актуальность. Статья посвящена разработке программных движений рабочего органа пропольщика в пространстве методом кинематического синтеза. Объект. Рабочий орган роботизированного пропольщика, в качестве которого используется фреза с вертикальной осью вращения. Материалы и методы. Решение задачи перемещения рабочего органа (фрезы) в пространстве подразделяется на два этапа: 1. Решается задача позиционирования манипуляционного механизма (задача конфигурации), соответствующего конечному положению в пространстве фрезы пропольщика, определяемого координатами сорняка исходя из критерия оптимизации; 2. После того как решена задача о конфигурации, ставится задача нахождения законов движения исполнительных звеньев по траектории. В работе рассмотрено безынерционное движение рабочего органа пропольщика по двум траектория: 1 - прямая; 2 - заранее неопределенная. Результаты и выводы. Решена задача перемещения рабочего органа (фрезы) пропольщика при движении по прямой и по предварительно неопределенной траектории по закону безынерционного движения с использованием оптимального алгоритма позиционирования. Сравнительный анализ перемещения рабочего органа пропольщика показал, что удлинение по неопределенной траектории рабочего органа незначительно (до $6 \%$ ), а также что при перемещении рабочего органа по прямой шток одного актуатора совершает возвратно-поступательное движение, что, в свою очередь, негативно сказывается на приводе, поэтому предпочтительней использовать предварительно неопределенную траекторию для перемещения рабочего органа пропольщика.

Ключевые слова: роботизированные пропольщики, технологический процесс прополки, рабочие органы пропольщика, исполнительные звенья, траектория перемещения, закон движения.

Цитирование. Иванов А. Г., Воробьева Н. С. Кинематика движения манипуляционного механизма роботизированного пропольщика при выполнении технологического процесса прополки. Известия НВ АУК. 2021. 4(64). 452-463. DOI: 10.32786/2071-9485-2021-04-44.

Авторский вклад. Все авторы настоящего исследования принимали непосредственное участие в планировании, выполнении или анализе данного исследования. Все авторы настоящей статьи одобрили представленный окончательный вариант.

Конфликт интересов. Авторы заявляют об отсутствии конфликта интересов.

Введение. При выращивании сельскохозяйственных культур возникает проблема борьбы с сорняками, которая является одной из трудоемких и энергозатратных сельскохозяйственных операций. Чтобы эффективно бороться с сорняками, необходимо знать физико-механические, физиологические характеристики сорняков, а также использовать эффективные методы борьбы и технические средства, применяемые для уничтожения сорняков.

Для решения проблемы полной прополки сорняков как в междурядьях, так и в рядках необходимо использование роботизированной сельскохозяйственной техники $[1,3,7]$, способной в автоматическом режиме удалять сорняки. За рубежом для решения проблемы сплошной прополки сорняков как в междурядьях, так и в рядках разработаны роботизированные машины и роботы $[10,12]$ (рисунки 1,2$)$. 


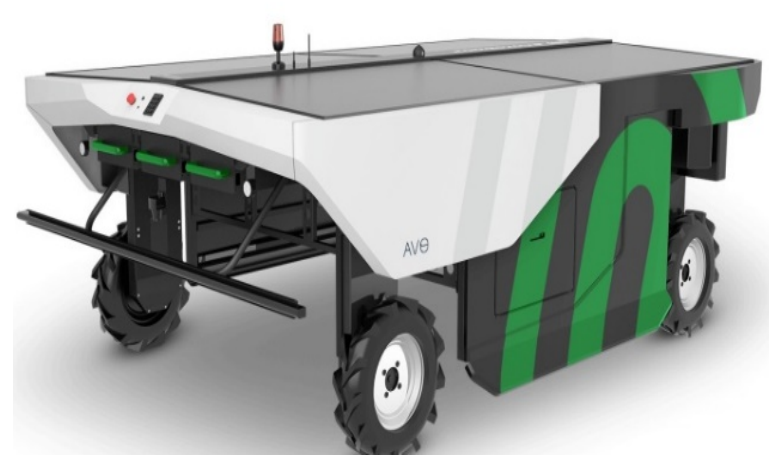

Рисунок 1 - Прополочный робот AVO

Figure 1 - AVO Weeding Robot

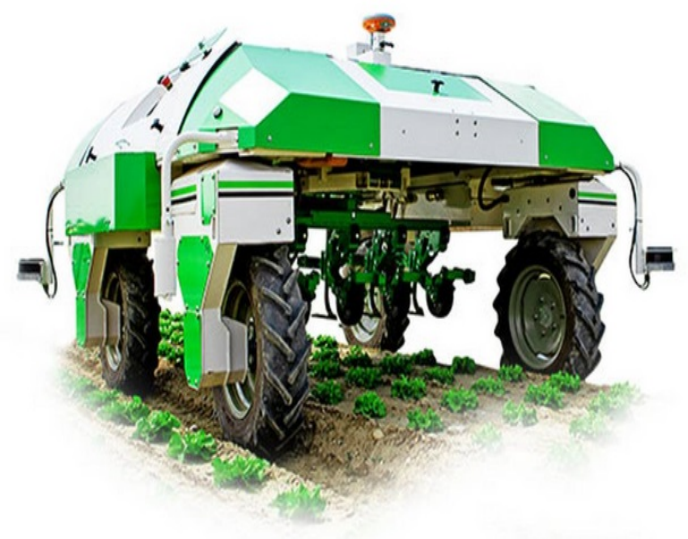

Рисунок 2 - Робот для удаления сорняков Dino

Figure 2 - Dino Weed Removal Robot

Но все они имеют ряд недостатков. Они изготовлены в единичных экземплярах, находятся в виде концептуальной модели или на стадиях доработки, готовые образцы зачастую имеют высокую стоимость. В России сельское хозяйство вообще отстаёт в разработке и использовании роботизированной техники от других стран и отраслей. Принципы и технические проблемы проектирования сельскохозяйственных роботизированных машин до конца не сформированы.

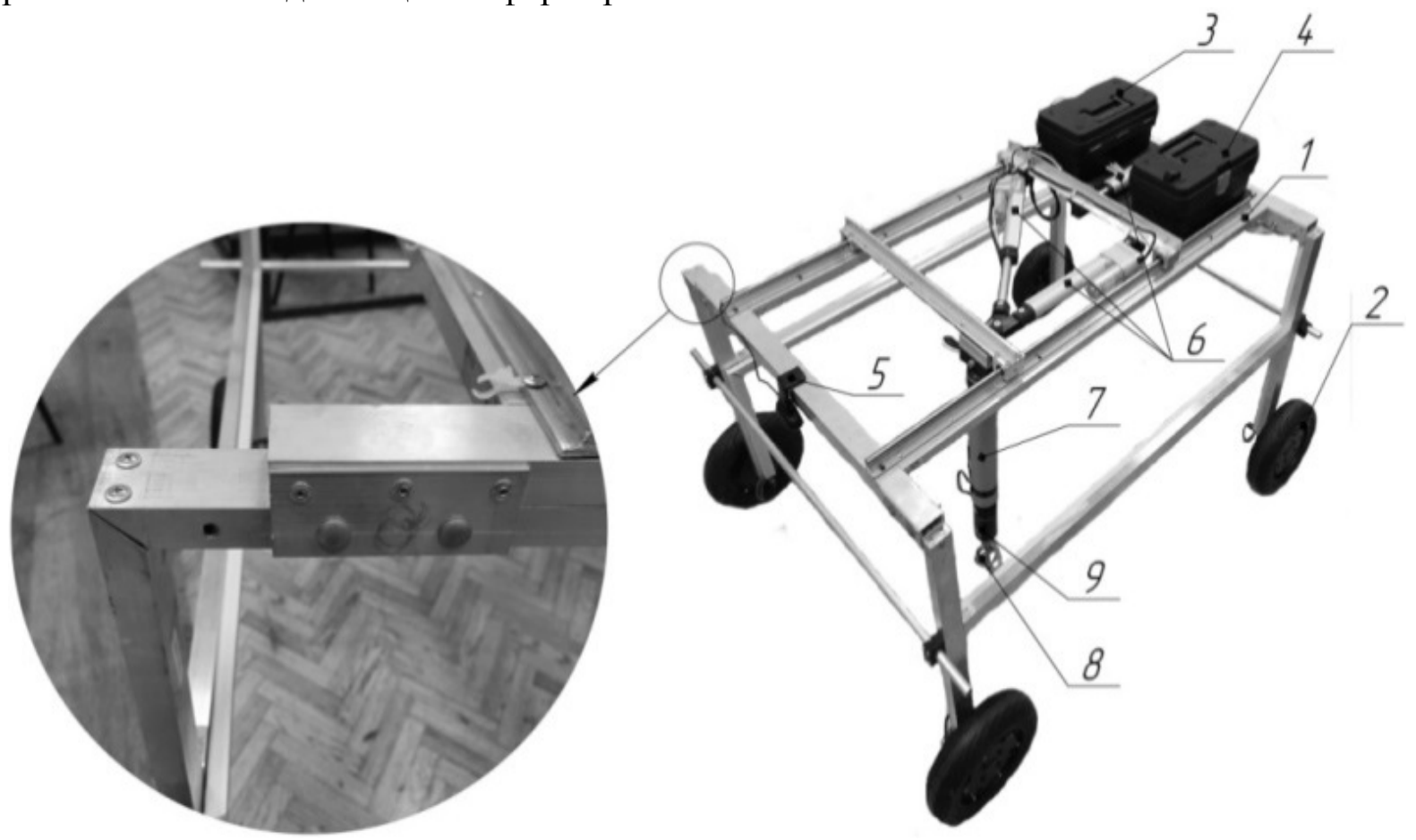

Рисунок 3 - Конструкция роботизированной прополочной машины: 1 - рама; 2 - приводные колеса; 3 - блок системы управления; 4 - бортовая система питания; 5 - камера СТ3;

6 - исполнительные приводы (актуаторы) 8 - рабочий орган (фреза); 9 - электродвигатель

Figure 3 - The design of the robotic weeding machine: 1 - frame; 2 - drive wheels; 3 - control system unit; 4 - on-board power supply system; 5 - STZ chamber; 6 - auxiliary drives (actuators) 8 - working body (milling cutter); 9 - electric motor 
Для решения существующих проблем разработан роботизированный пропольщик для механической борьбы с сорняками как в рядке, так и в междурядье (Роботпропольщик: пат. 2703092 Рос. Федерация. № 2019102103; заявл. 25.01.19; опубл. 15.10 .19 , Бюл. № 29. 8 с). Конструкция роботизированного пропольщика (рисунок 3) состоит из рамы 1 , мотора-колеса 2 , системы навигации и управления 3 , системы питания 4, датчика системы технического зрения 5, линейных приводов 6 и 7, осуществляющих перемещение рабочего органа 8 в пространстве, и электродвигателя 9, приводящего рабочий орган во вращательное движение [6,9].

В качестве рабочего органа пропольщика используется фреза с вертикальной осью вращения (рисунок 4). Обработка почвы фрезой обеспечивает интенсивное крошение, перемешивание, рыхление обрабатываемого слоя и уничтожение сорняков.

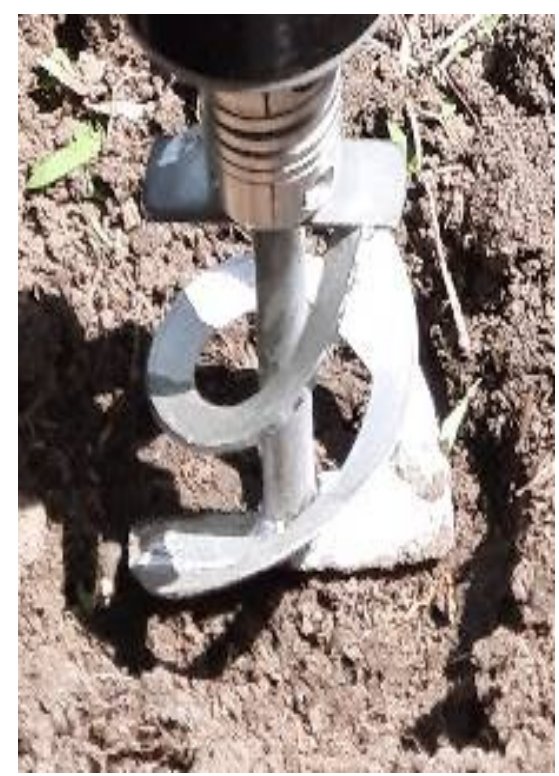

Рисунок 4 - Рабочий орган роботизированного пропольщика

Figure 4 - The working organ of the robotic weeding machine

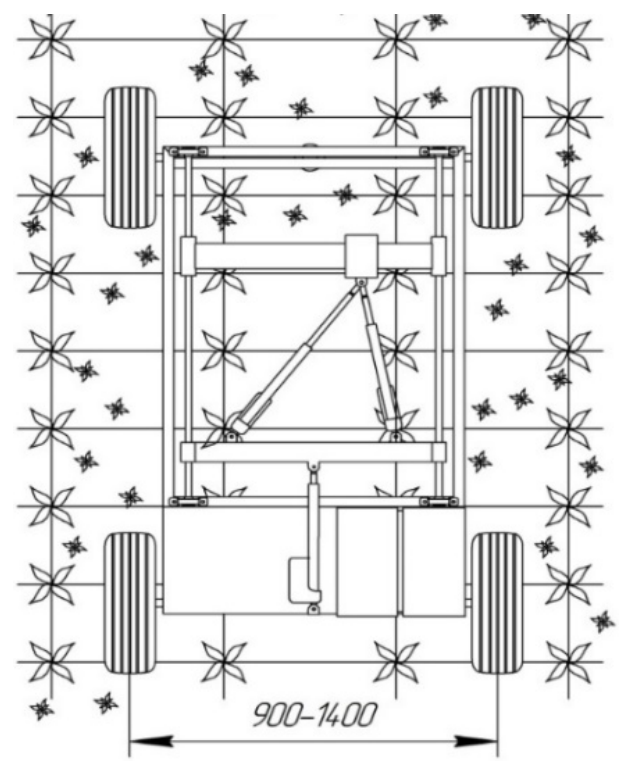

Рисунок 5 - Технологический процесс прополки с использование роботизированной прополочной машины

Figure 5 - Technological process of weeding with the use of a robotic weeding machine

К выращиванию сельскохозяйственных культур предъявляются различные агротехнологические требования: определенные размеры междурядья, междугнездья и др. Роботизированный пропольщик является универсальным, поэтому его конструкция предусматривает изменение колеи колес от 900 до 1400 мм с интервалом 50 мм под необходимое междурядье за счет раздвижного механизма (рисунок 3 ).

Технологический процесс прополки с использованием роботизированного пропольщика осуществляется по этапам (рисунки 5, 6). На первом этапе строится карта перемещения пропольщика на местности, на следующем этапе - шасси пропольщика в автоматическом режиме, за счет системы навигации и управления, заезжает в рядки с растениями, затем с помощью системы технического зрения определяются координаты сорных растений и рассчитывается оптимальный путь перемещения рабочего органа между сорняками, на последнем этапе рабочий орган в автоматическом режиме перемещается к сорняку, срезает его и взрыхляет почву в окрестностях сорняка. 


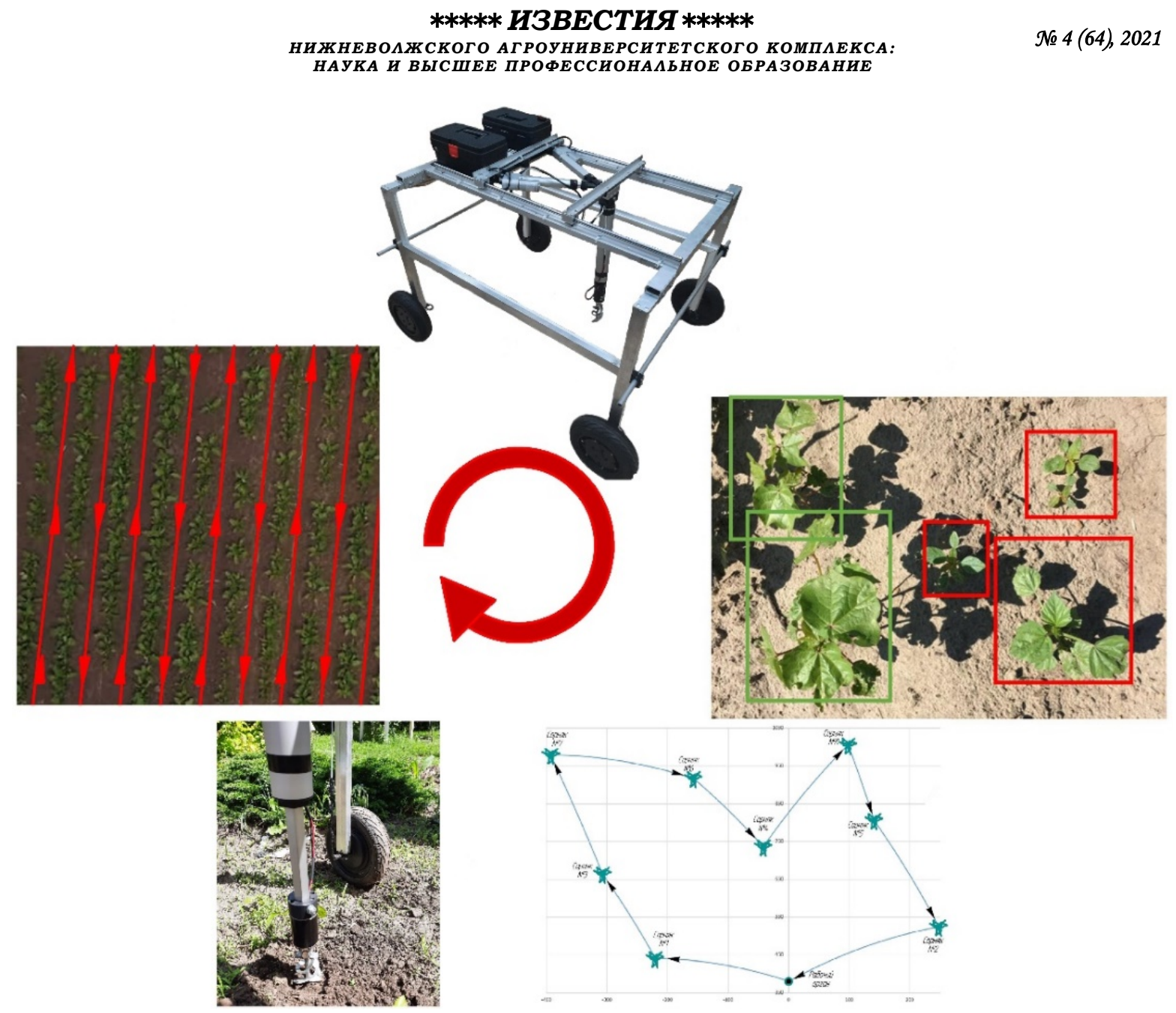

Рисунок 6 - Принцип работы роботизированного пропольщика

Figure 6 - The principle of operation of the robotic weeding machine

Материалы и методы. Для осуществления всех этапов приведенного технологического процесса необходимо решить ряд задач. Одной из них является задача программного перемещения рабочего органа, роботизированного пропольщика в пространстве методом кинематического синтеза $[8,13,14]$.

Задача перемещения рабочего органа (фрезы) в пространстве подразделяется на два этапа:

1. Решение задачи позиционирования манипуляционного механизма (задача о конфигурации), соответствующего конечному положению в пространстве фрезы пропольщика, определяемого координатами сорняка. Задача носит оптимизационный характер.

2. Синтез законов перемещения по траектории в пределах зоны обслуживания.

Перемещение рабочего органа пропольщика заключается в необходимости передвижения фрезы из нулевого (начального) положения, имеющего координаты $M_{0}\left(X_{M 0}, Y_{M 0}\right)$, в заданное конечное положение $M_{\kappa}\left(X_{M \kappa}, Y_{M \kappa}\right)$, и которые не выходят за пределы рабочей зоны (рисунок 7) [11].

В нулевом положении длины актуаторов, принятые за обобщённые координаты $l_{i 0}$, известны по показаниям датчиков положения (потенциометров). Поэтому ставится задача определения длин актуаторов, принятых за обобщенные координаты $l_{i \kappa}$, соответствующие конечному положению фрезы пропольщика (рисунок 8) [2, 4]. 


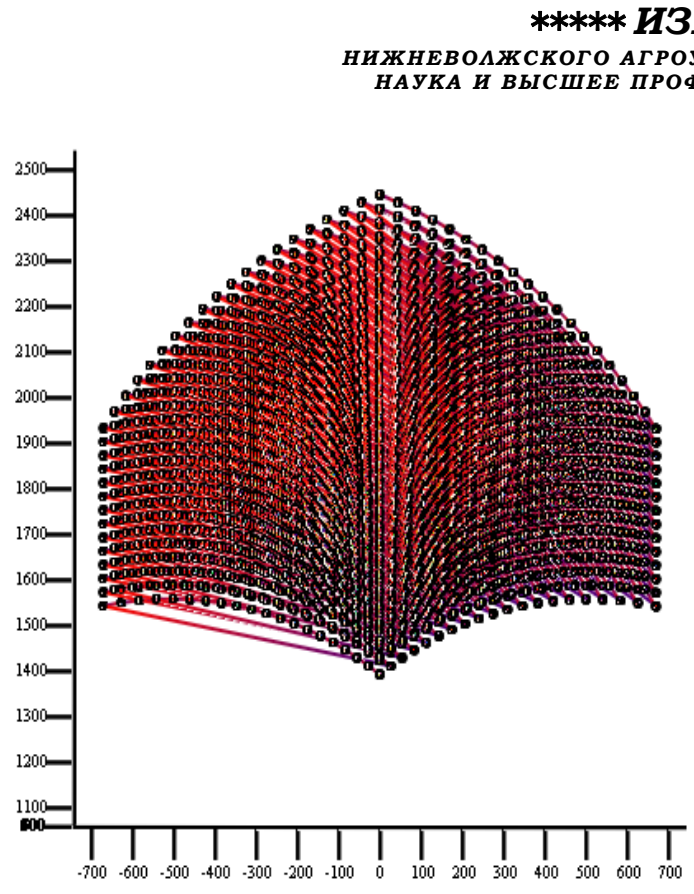

Рисунок 7 - Рациональная рабочая зона роботизированного пропольщика

Figure 7 - Rational working area of a robotic weeding machine

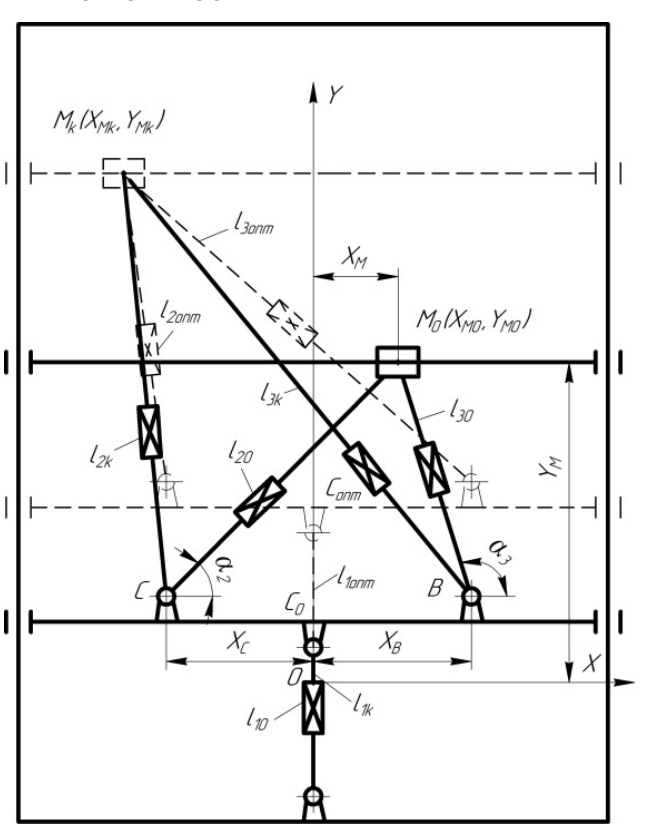

Рисунок 8 - Схема перемещения фрезы из нулевого положения в конечное

(плоскость XOY)

Figure 8 - Diagram of moving the milling cutter from the zero position to the final one

(XOY plane)

Длина звена $l_{1 \mathrm{k}}$ находится с учетом критерия энергоэффективности - минимизации квадратичной функции, характеризующей изменения длин звеньев манипуляционного механизма (1), а длины звеньев $l_{2 \mathrm{k}}, l_{3 \mathrm{k}}$ определяются из выражений (2):

$$
\begin{aligned}
& \Phi_{l k \text { min }}=C_{1} \cdot\left(l_{1 k}-l_{10}\right)^{2}+C_{2} \cdot\left[\sqrt{\left(Y_{M k}-l_{1 k}\right)^{2}+\left(X_{C}+X_{M k}\right)^{2}}-\sqrt{\left(Y_{M 0}-l_{10}\right)^{2}+\left(X_{C}+X_{M 0}\right)^{2}}\right]^{2}+ \\
& +C_{3} \cdot\left[\sqrt{\left(Y_{M k}-l_{1 k}\right)^{2}+\left(X_{B}-X_{M k}\right)^{2}}-\sqrt{\left(Y_{M 0}-l_{10}\right)^{2}+\left(X_{B}-X_{M 0}\right)^{2}}\right]^{2},
\end{aligned}
$$

где $l_{10}$ и $l_{1 k}$ - начальная и конечная длина 1-го звена, мм; $\mathrm{C}_{1}, \mathrm{C}_{2}, \mathrm{C}_{3}-$ весовые коэффициенты; $X_{C}=X_{B}=a / 2$ - постоянные, в выбранной системе, координаты точек крепления звеньев манипуляционного механизма.

Координаты точки $M$ и длины исполнительных звеньев робота в системе координат $O X Y$ имеют вид [5]:

$$
\left\{\begin{array}{l}
\left(Y_{M}-l_{1}\right)^{2}+\left(X_{M}-X_{B}\right)^{2}=l_{2}^{2}, \\
\left(Y_{M}-l_{1}\right)^{2}+\left(X_{M}+X_{B}\right)^{2}=l_{3}^{2} .
\end{array}\right.
$$

где $X_{M}, Y_{M}$ - координаты точки $M$ в неподвижной системе координат.

После того как решена задача позиционирования рабочего органа манипулятора, ставится задача нахождения законов движения исполнительных звеньев $l_{i}(t)$ по траектории. Данная задача может решаться выбором для каждого исполнительного звена одного из распространенных законов движения, удовлетворяющих граничным условиям [11]: 


$$
\begin{aligned}
& \text { ***** НЗВЕСТИЯ ***** } \\
& \text { НИЖНЕВОАЖСКОГО АГРОУНИВЕРСИТЕТСКОГО КОМПАЕКСА: } \\
& \text { НАУКА И ВЫСШЕЕ ПРОФЕССИОНАМЬНОЕ ОБРАЗОВАНИЕ } \\
& \begin{array}{l}
l_{i k}(0)=l_{i 0}, \quad l_{i k}\left(t_{k}\right)=l_{i k}, \quad i=1,2,3 \\
l_{i k}(0)=0, \quad i_{i k}\left(t_{k}\right)=0
\end{array}
\end{aligned}
$$

Зная начальные длины актуаторов $l_{10}, l_{20}, l_{30}$, и конечную конфигурацию манипуляционного механизма $l_{1 k}, l_{2 k}, l_{3 k}$, определенную из уравнения (1), выберем закон движения штоков актуаторов от $l_{i 0}$ до $l_{i k}$, обеспечивающий нулевые значения скоростей, ускорений в момент трогания и останова, т.е. безынерционное движение рабочего органа.

Данному требованию удовлетворяют следующие законы движения: синусоидальный, полиномиальный, трапецеидальный. Воспользуемся синусоидальным законом:

$$
l(t)=l_{0}+\left(l_{k}-l_{0}\right) \cdot\left[\frac{t}{T}-\frac{1}{2 \pi} \sin \left(\frac{2 \pi t}{T}\right)\right],
$$

где $l_{0}$ - длина актуатора в начальном положении, м; $l_{k}$ - длина актуатора в конечном положении, м; $t$ - время в текущий момент движения актуатора, с; $T$ - время, за которое актуатор переместится из начального положения в конечное.

Законы изменения длин исполнительных звеньев рассматриваются при движении рабочего органа в двух случаях: траектория заранее не определена и траекторией перемещения по времени является прямая.

Время Т зависит от скорости актуаторов, так как изменение длин звеньев $\left(l_{i k}-\right.$ $\left.l_{i 0}\right)$ различны, то и время $\mathrm{T}$ для всех актуаторов будет также различно. Для решения задач управления принимаем время $T=\max _{i=1,2,3}\left(T_{i}\right)$, т.е. одинаковое для всех актуаторов.

Используя законы изменения длин актуаторов (4) и зависимости координат точки М от длин актуаторов [11], получим закон изменения координат по времени:

$$
\left\{\begin{array}{l}
X_{M}(t)=-\frac{l_{3}(t)^{2}-l_{2}(t)^{2}}{4 X_{B}}, \\
Y_{M}(t)=l_{1}(t)+\sqrt{l_{2}(t)^{2}-\left(\frac{l_{3}(t)^{2}-l_{2}(t)^{2}}{4 X_{B}}\right)^{2}+\frac{l_{3}(t)^{2}-l_{2}(t)^{2}}{2}-X_{B}^{2}} .
\end{array}\right.
$$

Оптимальной траекторией перемещения по времени считается прямая, в этом случае задача позиционирования сводится к перемещению рабочего органа по отрезку $\mathrm{M}_{0} \mathrm{M}_{\mathrm{k}}$. Канонические уравнения прямой в плоскости, проходящей через две точки, имеют следующий вид:

$$
\frac{X_{M}-X_{M 0}}{\Delta X}=\frac{Y_{M}-Y_{M 0}}{\Delta Y},
$$

где $\Delta X=X_{M \mathrm{k}}-X_{M 0}, \Delta Y=Y_{M \mathrm{k}}-Y_{M 0}$.

Из уравнения 6 выразим зависимости $X_{M}, Y_{M}$, составим систему уравнений, примем обозначение констант, зависящих от начальных и конечных координат рабочего органа:

$$
K_{1}=\frac{\Delta X}{\Delta Y}, b_{1}=-K_{1} Y_{M 0}+X_{M 0}, b_{2}=-\frac{X_{M 0}}{K_{1}}+Y_{M 0}
$$

В результате, имеем: 
Длина элементарного участка кривой имеет вид:

$$
d S=\sqrt{d X_{M}^{2}+d Y_{M}^{2}} .
$$

Используя систему уравнений (8), определим выражение (9):

$$
\left\{\begin{array}{l}
d S=\sqrt{1+1 / K_{1}^{2}} d X_{M} \\
d S=\sqrt{1+K_{1}^{2}} d Y_{M}
\end{array} .\right.
$$

Проинтегрируем систему уравнений $(10)$ и определим зависимости $\left.l_{i}(S)\right)$ :

$$
\left\{\begin{array}{l}
S=\sqrt{1+1 / K_{1}^{2}} \cdot X_{M}+C_{1} \\
S=\sqrt{1+K_{1}^{2}} \cdot Y_{M}+C_{2}
\end{array} .\right.
$$

Определим $X_{M}, Y_{M}$, для этого введём обозначения коэффициентов $K \mathrm{x}, K \mathrm{y}$ :

$$
\left\{\begin{array}{l}
K_{X}=\sqrt{1+1 / K_{1}^{2}}, \\
K_{Y}=\sqrt{1+K_{1}^{2}},
\end{array}\right.
$$

и определим постоянные $C_{1}, C_{2}$, при начальных условиях $X_{M 0}, Y_{M 0}$, и $S_{0}=0$ :

$$
\left\{\begin{array}{l}
C_{1}=-X_{M 0} \cdot K_{X}, \\
C_{2}=-Y_{M 0} \cdot K_{Y},
\end{array}\right.
$$

Из выражений (8) с учётом (12), (13) получим:

$$
X_{M}=\frac{S+X_{M 0} K_{X}}{K_{X}}, Y_{M}=\frac{S+Y_{M 0} K_{Y}}{K_{Y}} .
$$

Закон движения рабочего органа по прямой траектории примем по закону безынерционного движения рабочего органа:

$$
S(t)=S_{k} \cdot\left[\frac{t}{T}-\frac{1}{2 \pi} \sin \left(\frac{2 \pi t}{T}\right)\right] .
$$

Тогда получаем зависимость перемещения рабочего органа (точки М) от времени с учетом выбранного закона движения (15):

$$
\begin{aligned}
& X_{M}(t)=\frac{S(t)+X_{M 0} K_{X}}{K_{X}}, \\
& Y_{M}(t)=\frac{S(t)+Y_{M 0} K_{Y}}{K_{Y}} .
\end{aligned}
$$

Таким образом, решая уравнения (2) с учетов зависимостей (16), определим законы изменения длин исполнительных звеньев $l_{i}(t)$ : 
где $X_{B}$ - координаты крепления звена $l_{2}$ на раме в абсолютной системе.

Результаты и обсуждение. На рисунках 9 - 11 приведены сравнительные графики изменения длин актуаторов при перемещении рабочего органа из точки А в точку В (рисунок 12) по заранее неопределенной и прямой траекториям.

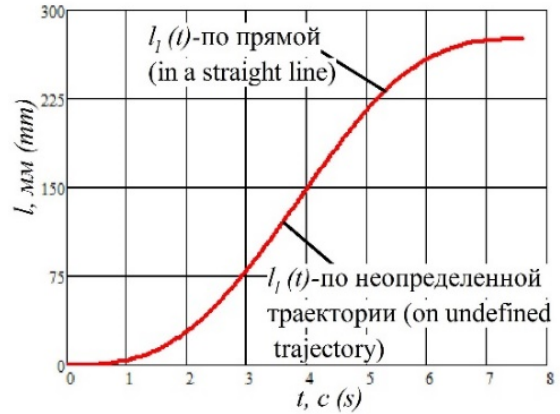

Рисунок 9 - Изменение длины актуатора $l_{1}$ от времени

Figure 9 - Change in length actuator $l_{1}$ from time to time

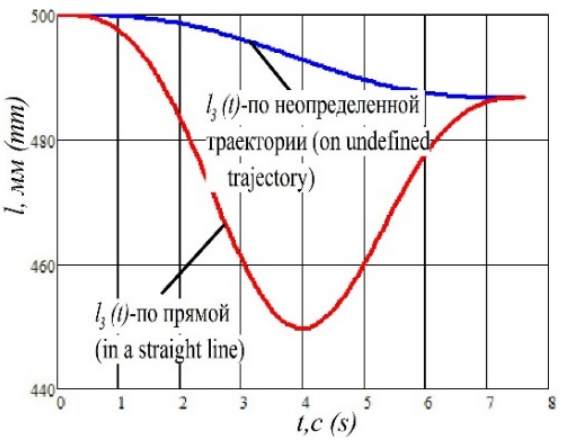

Рисунок 11 - Изменение длины актуатора $l_{3}$ от времени

Figure 11 - Change in length actuator $l_{3}$ from time to time

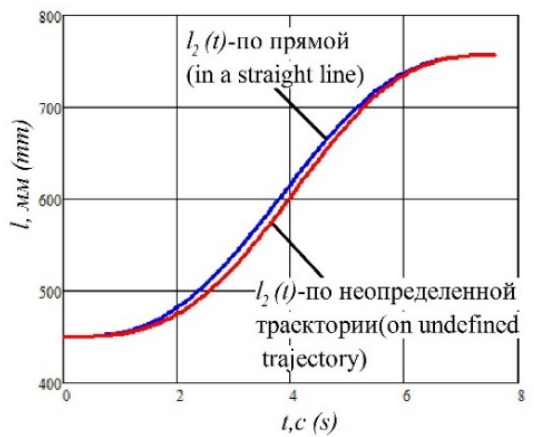

Рисунок 10 - Изменение длины актуатора $l_{2}$ от времени Figure 10 - Change in length actuator $l_{2}$ from time to time

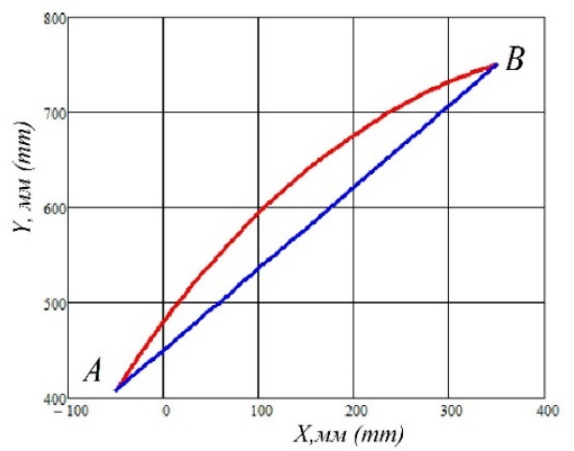

Рисунок 12 - Траектории перемещения рабочего органа в плоскости XOY

Figure 12 - Trajectory of movement of the working body in the XOY plane.

Решение задачи перемещения рабочего органа пропольщика по прямой и по заранее неопределенной траекториям в начальной и конечной точках имеет одинаковый результат (рисунок 12), что говорит о правильности математической модели.

На графике (рисунок 13) приведены зависимости максимального отклонения рабочего органа пропольщика от прямой $\left(h_{\max }\right)$ и удлинение заранее неизвестной траектории $(\Delta S)$ в зависимости от длины прямолинейной траектории.

Сравнительный анализ перемещения рабочего органа пропольщика (рисунок 10) показал, что если длина траектории по прямой не превышает 250 мм, то удлинение по неопределенной траектории рабочего органа $(\Delta S)$ не более $2 \%$, а наибольшее отклонение рабочего органа пропольщика от прямой менее $5 \%$. Если длина траектории по прямой более $250 \mathrm{Mm}$, то $\Delta \mathrm{S}$ не превышает $6 \%$, a $\mathrm{h}_{\max }$ не превышает $14 \%$. 


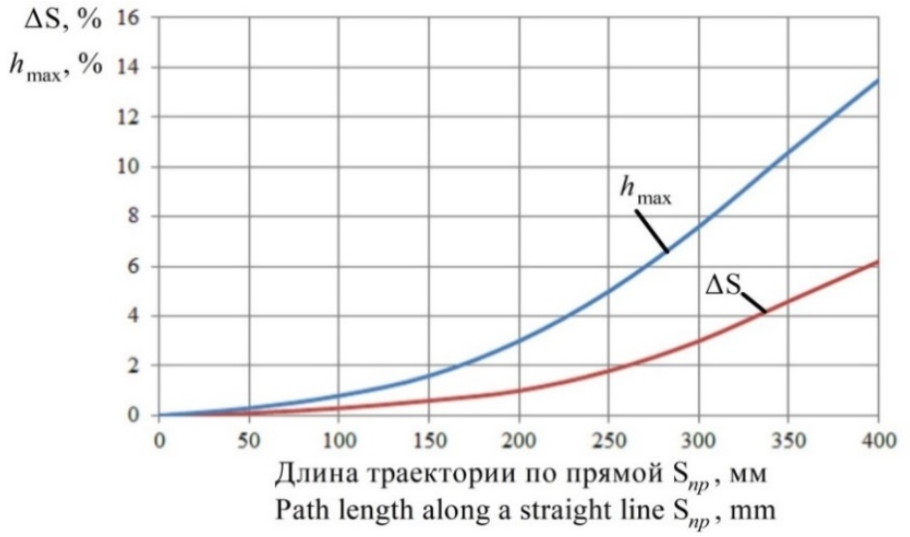

Рисунок 13 - Зависимости максимального отклонения рабочего органа пропольщика от прямой $\left(h_{\max }\right)$ и удлинение заранее неизвестной траектории $(\Delta \mathrm{S})$ в зависимости от длины прямолинейной траектории

Figure 13 - Dependences of the maximum deviation of the weeding tool from the straight line $\left(h_{\max }\right)$ and the elongation of the previously unknown trajectory $(\Delta \mathrm{S})$ depending on the length of the straight trajectory

Полученные графики изменения скоростей штоков исполнительных звеньев (актуаторов) при перемещении рабочего органа по прямой и неопределенной траектории (рисунок 14).

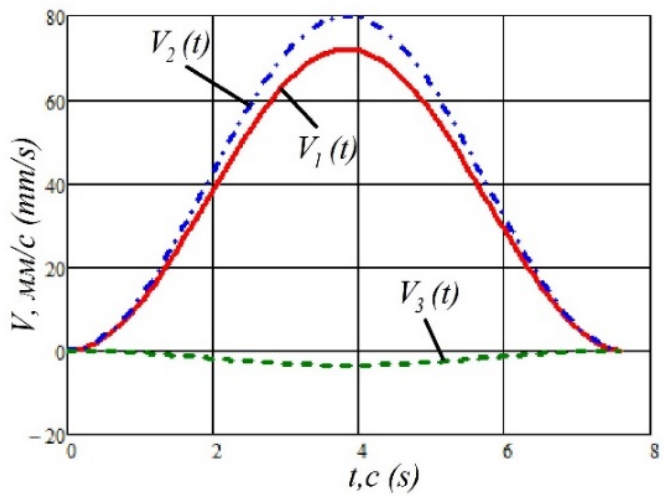

a)

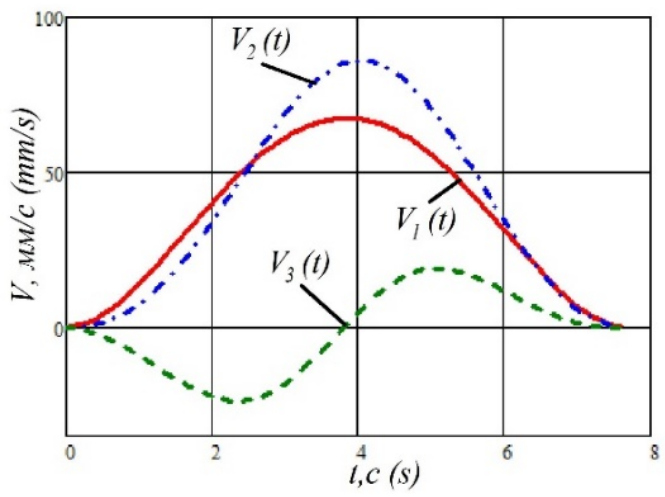

б)

Рисунок 14 - График изменения скоростей штоков исполнительных звеньев (актуаторов) при перемещении рабочего органа: а) по неопределенной траектории; б) по прямой

Figure 14 - Graph of changes in the speeds of the rods of the executive links (actuators) when moving the working body: a) along an undefined trajectory; b) in a straight line

Выводы. При перемещении рабочего органа по прямой траектории линейная скорость одного из исполнительных звеньев меняет знак, это говорит о том, что шток актуатора совершает возвратно-поступательное движение, что, в свою очередь, негативно сказывается на приводе. Таким образом, несмотря на то что длина пути по заранее неопределенной траектории больше на 1-6 \%, предпочтительней использовать данную траекторию для перемещения рабочего органа пропольщика.

\section{Библиографический список}

1. Абросимов В. К., Елисеев В. В. Малые интеллектуальные роботы для решения задач точного земледелия: проблемы и решение // Робототехника и техническая кибернетика. 2018. № 4 (21). C. 14-18. 


\section{***** H3BECTYЯ ***** \\ НИЖНЕВОАЖСКОГО АГРОУНИВЕРСИТЕТСКОГО КОМПАЕКСА: НАУКА И ВЫСШЕЕ ПРОФЕССИОНААЬНОЕ ОБРАЗОВАНИЕ}

2. Вирабян Л. Г., Халапян С. Ю., Кузьмина В. С. Оптимизация траектории позиционирования выходного звена планарного параллельного робота // Вестник БГТУ им. В. Г. Шухова. 2018. № 9. С. 106-113.

3. Годжаев 3. А. Тенденции развития средств механотроники, автоматизации и роботизации для синтеза новых высокопроизводительных технологий, и машин в сельском хозяйстве // Фундаментальные и прикладные проблемы техники и технологии. 2018. № 3 (329). C. 4-16.

4. Жога В. В. Задача позиционирования манипулятора параллельно-последовательной структуры с управляемым захватным устройством // Мехатроника, автоматизация, управление. 2016. № 8. Том 17. С. 525-530.

5. Жога В. В. Система управления манипулятора - трипода // Робототехника и техническая кибернетика. 2014. № 4 (5). С. 58-62.

6. Иванов А. Г., Воробьева Н. С., Дяшкин А. В. Обоснование геометрических параметров робота-пропольщика. // Известия Волгоградского государственного технического университета. 2019. № 3 (226). С. 22-25.

7. Florance Mary M., Yogaraman D. (2021). Neural network based weeding robot for crop and weed discrimination // Paper presented at the Journal of Physics: Conference Series. 2021. № 1979 (1).

8. Grimstad L. Kinematic modeling and control design of a novel single-rail parallel arm // Paper presented at the IFAC-PapersOnLine. 2017. № 50 (1). P. 11434-11440.

9. Ivanov A. G. Dynamic model of end-effector actuator used for mobile robotic weeder // Paper presented at the IOP Conference Series: Materials Science and Engineering. 2020. № 747 (1).

10. Machleb J. Sensor-based intrarow mechanical weed control in sugar beets with motorized finger weeders. Agronomy. 2021. № 11 (8).

11. Ovchinnikov A. S. Kinematic study of the weeding robot. Paper presented at the IOP Conference Series: Materials Science and Engineering. 2019. № 489 (1).

12. Raja R., Nguyen T. T. RTD-SEPs: Real-time detection of stem emerging points and classification of crop-weed for robotic weed control in producing tomato // Biosystems Engineering. 2020. № 195. P. 152-171.

13. Roshanianfard A., Mengmeng D., Nematzadeh S. A 4-DOF SCARA robotic arm for various farm applications: Designing, kinematic modelling, and parameterization // Acta Technologica Agriculturae. 2021. № 24 (2). P. 61-66.

14. Xuelei W., Jie H. Kinematics and statics analysis of a novle 4-dof parallel mechanism for laser weeding robot // INMATEH - Agricultural Engineering. 2016. № 50 (3). P. 29-38.

Conclusion. When moving the working body along a straight trajectory, the linear velocity of one of the executive links changes sign, this indicates that the actuator rod performs a reciprocating motion, which in turn negatively affects the drive. Thus, despite the fact that the length of the path along a predetermined trajectory is $1-6 \%$ longer, it is preferable to use this trajectory to move the working organ of the weaver.

\section{References}

1. Abrosimov V. K., Eliseev V. V. Small intelligent robots for solving precision farming problems: problems and solutions // Robotics and technical cybernetics. 2018. No. 4 (21). P. 14-18.

2. Virabyan L. G., Khalapyan S. Y., Kuzmina V. S. Optimization of the positioning trajectory of planar parallel robot output link // Bulletin of BSTU named after V. G. Shukhov. 2018. № 9. P. 106-113.

3. Gojaev Z. A. Trends in the development of mechanotronics, automation and robotization tools for the synthesis of new high-performance technologies, and machines in agriculture // Fundamental and applied problems of engineering and technology. 2018. № 3 (329). Pp. 4-16.

4. Zhoga V. V. The Problem of positioning a manipulator of a parallel-sequential structure with a controlled gripper device // Mechatronics, automation, control. 2016. No. 8. V. 17. P. 525-530. 


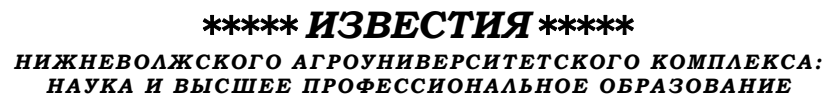

№ 4 (64), 2021

НАУКА И ВЫСШЕЕ ПРОФЕССИОНААЬНОЕ ОБРАЗОВАНИЕ

5. Zhoga V. V. Control system of the manipulator - tripod // Robotics and technical cybernetics. 2014. No. 4 (5). P. 58-62.

6. Ivanov A. G., Vorob'yeva N. S., Dyashkin A. V. Substantiation of geometric parameters of the weeding robot // News of the Volgograd State Technical University. 2019. No. 3 (226). P. 22-25.

7. Florance Mary M., Yogaraman D. (2021). Neural network based weeding robot for crop and weed discrimination // Paper presented at the Journal of Physics: Conference Series. 2021. № 1979 (1).

8. Grimstad L. Kinematic modeling and control design of a novel single-rail parallel arm // Paper presented at the IFAC-PapersOnLine. 2017. № 50 (1). P. 11434-11440.

9. Ivanov A. G. Dynamic model of end-effector actuator used for mobile robotic weeder // Paper presented at the IOP Conference Series: Materials Science and Engineering. 2020. № 747 (1).

10. Machleb J. Sensor-based intrarow mechanical weed control in sugar beets with motorized finger weeders. Agronomy. 2021. № 11 (8).

11. Ovchinnikov A. S. Kinematic study of the weeding robot. Paper presented at the IOP Conference Series: Materials Science and Engineering. 2019. № 489 (1).

12. Raja R., Nguyen T. T. RTD-SEPs: Real-time detection of stem emerging points and classification of crop-weed for robotic weed control in producing tomato // Biosystems Engineering. 2020. № 195. P. 152-171.

13. Roshanianfard A., Mengmeng D., Nematzadeh S. A 4-DOF SCARA robotic arm for various farm applications: Designing, kinematic modelling, and parameterization // Acta Technologica Agriculturae. 2021. № 24 (2). P. 61-66.

14. Xuelei W., Jie H. Kinematics and statics analysis of a novle 4-dof parallel mechanism for laser weeding robot // INMATEH - Agricultural Engineering. 2016. № 50 (3). P. 29-38.

\section{Author's Information}

Ivanov Aleksey Gennadievich, assistant of the department "Mechanics" Volgograd State Agrarian University (400002, Volgograd, Universitetsky Prospekt 26), tel. +7 (8442) 41-18-49. E-mail: leha_2106@list.ru

Vorob'eva Natalia Sergeevna, head of the department "Mechanics" Volgograd State Agrarian University (400002, Volgograd, Universitetsky Prospekt 26), candidate of technical sciences, tel. +7 (8442) 41-18-49.

\section{Информация об авторах}

Иванов Алексей Геннадьевич, ассистент кафедры «Механика» ФГБОУ ВО «Волгоградский государственный аграрный университет» (400002, г. Волгоград, Университетский проспект, 26), тел. +7 (8442) 41-18-49. E-mail: leha_2106@list.ru ORCID 0000-0003-1098-099X

Воробьева Наталья Сергеевна, заведующий кафедрой «Механика» ФГБОУ ВО «Волгоградский государственный аграрный университет» (400002, г. Волгоград, Университетский проспект, 26), кандидат технических наук, тел. +7 (8442) 41-18-49. E-mail: vgsxa@mail.ru ORCID 0000-0001-9035-6382

\author{
V.S. Bocharnikov ${ }^{1}$, A.A. Borovikov ${ }^{2}$ \\ ${ }^{1}$ Volgograd State Agrarian University, Volgograd \\ ${ }^{2}$ Belarusian State Agricultural Academy, Gorki
}

EFFICIENCY OF ANTI-FILTRATION SAND SAPROPEL CURTAIN ON THE EXAMPLE OF THE GATEWAY-REGULATOR OF RECLAMATION SYSTEMS

Received 18.09.2021

Submitted 20.11.2021

\section{Summary}

The article examines the existing and prospective designs of the anti-seepage device, compares their anti-seepage efficiency using the method of resistance coefficients of R.R. Chugaev. Comparison of the costs for the construction of devices is made, taking into account the cost of the required materials. The economic effect of the introduction has been calculated. 